\title{
Erratum to: Russula chiui and R. pseudopectinatoides, two new species from southwestern China supported by morphological and molecular evidence
}

\author{
Guo-Jie $\mathrm{Li}^{1}$ • Dong Zhao ${ }^{1} \cdot$ Sai-Fei $\mathrm{Li}^{1,2} \cdot$ Hua-An Wen ${ }^{1}$
}

Published online: 25 June 2015

(C) German Mycological Society and Springer-Verlag Berlin Heidelberg 2015

Erratum to: Mycol Progress (2015) 14:33

DOI 10.1007/s11557-015-1054-y

The original version of this article inadvertently contained mistake. The authors have just found that the order of the plates and the figures are not correct. See below for the correct presentation:

Plate 1 should be the colored plate which contains the fruiting bodies and the SEM photos (current Fig 3). Fig 2 should be the current Plate 1, and Fig 3 should be the current Fig 2.

The online version of the original article can be found at http://dx.doi.org/ 10.1007/s11557-015-1054-y.

Hua-An Wen

wenha@im.ac.cn

Guo-Jie Li

liguojie.hbu@gmail.com

Dong Zhao

miczhd@163.com

Sai-Fei Li

flylee0118@163.com

1 State Key Laboratory of Mycology, Institute of Microbiology, Chinese Academy of Sciences, No 1 Beichen West Road, Chaoyang, District, Beijing 100101, China

2 University of Chinese Academy of Sciences, No.19AYuquan Road, Shijingshan District, Beijing 100049, China 